\title{
Esthétique du mensonge
}

Ästhetik der Lüge

Aesthetics of lying

Jochen Mecke

\section{(2) OpenEdition}

\section{Journals}

Édition électronique

URL : http://journals.openedition.org/ceg/1436

DOI : $10.4000 /$ ceg. 1436

ISSN : 2605-8359

\section{Éditeur}

Presses Universitaires de Provence

Édition imprimée

Date de publication : 15 juin 2015

Pagination : 76-91

ISBN : 978-2-85399-993-9

ISSN : 0751-4239

\section{Référence électronique}

Jochen Mecke, «Esthétique du mensonge », Cahiers d'Études Germaniques [En ligne], 68 | 2015, mis en ligne le 17 décembre 2017, consulté le 01 décembre 2020. URL : http://journals.openedition.org/ceg/ 1436 ; DOI : https://doi.org/10.4000/ceg.1436 


\section{Esthétique du mensonge}

Jochen MECKE

Université de Regensburg

\section{La littérature - un art incapable de mentir?}

Si nous essayons de faire abstraction de la longue tradition de la condamnation catégorique du mensonge - une puissante tradition fondée par saint Augustin, refondée par Immanuel Kant et qui détermine toujours le catéchisme catholique ainsi que l'éducation laïque des enfants - et de nous abstenir également de tout jugement moral, pour nous baser sur ce qui peut être considéré comme le plus petit dénominateur commun de toutes les définitions du mensonge, nous pouvons en établir les éléments suivants: 1 . Tout mensonge consiste en une divergence entre un sentiment ou une opinion d'une part et une énonciation ou une expression d'autre part. 2. Cette divergence est dissimulée. 3. Elle sert à des objectifs qui restent en général également dissimulés ${ }^{1}$.

De cette définition s'ensuit que la littérature n'est point capable de mentir au sens propre du terme. Car si, bien sûr, elle fait bel et bien montre d'une divergence entre opinion et expression - puisque l'auteur feint de croire à une histoire qu'il a simplement inventée - elle ne remplit pourtant point la deuxième condition, car cette divergence n'est pas du tout dissimulée mais connue de chaque lecteur, dès qu'il ouvre un livre. C'est évidemment le code littéraire même qui signale au lecteur qu'il s'agit d'une fiction et qui révèle donc le "mensonge" littéraire. Or, tout mensonge qui se révèle lui-même comme tel n'en est plus un ${ }^{2}$. Toute œuvre littéraire s'inscrit d'emblée dans un «pacte fictionnel » qui inclut la suspension of disbelief, c'est-à-dire qui permet au lecteur de suspendre tout soupçon de mensonge et toute incrédulité, ce qui est une partie intégrante et structurelle du pacte littéraire ${ }^{3}$.

\footnotetext{
1 Cf. Simone Dietz, Die Kunst des Lügens. Eine sprachliche Fähigkeit und ihr moralischer Wert, Reinbek. Hamburg, Rowohlt, 2003, p. 25.

2 « Kunst behandelt den Schein als Schein, will also gerade nicht täuschen, ist wahr » (Friedrich Nietzsche, Kritische Studienausgabe in 15 Bänden, éd. par Giorgio Colli et Mazzino Montinari, München, dtv, 1999, Nachgelassene Fragmente 1869-1874, p. 632).

3 Le terme consacré dans la théorie littéraire a été forgé par Coleridge. Il décrit l'attitude d'un lecteur averti, qui consiste à "croire" à la réalité du monde de la fiction, même s'il contient des éléments fantastiques (cf. Samuel Taylor Coleridge, Biographia Literaria, t. II, Oxford, Clarendon Press, 1907, p. 6).
} 
Il est d'ailleurs significatif que le premier roman moderne mette en scène un lecteur qui est incapable de décoder les signes du "mensonge" et du pacte de la fiction, ce qui fait qu'il prend les œuvres littéraires au pied de la lettre pour les confondre avec la réalité. Don Quichotte peut et veut croire que les histoires qu'il a lues dans les livres de chevalerie sont réelles. De cette manière, Cervantès ridiculise la confusion entre littérature et mensonge. Il répond également de façon implicite au reproche de Platon pour s'en moquer. Par une mise en abyme, l'auteur introduit le reproche de mensonge comme un vieux préjugé contre la littérature dans le Don Quichotte même. Car dans le chapitre peut-être le plus célèbre du roman qui relate entre autres le combat contre les moulins à vent, le récit s'interrompt de manière abrupte, parce que le manuscrit dont dispose l'auteur fictif s'arrête à cet endroit précis ${ }^{4}$. Or, dans ses recherches, le «premier auteur» trouve par hasard à Tolède un manuscrit où un auteur arabe nommé Cide Hamete Benengeli raconte la suite des aventures de Don Quichotte. Mais, comme l'auteur le souligne malicieusement, le fait que dorénavant les prouesses du héros sont racontées par un Arabe met le lecteur face à un problème grave, car les Arabes - comme tout le monde le sait - ont la réputation bien ancrée de mentir. Toutefois, comme le " premier auteur » le souligne, s'il est certain que les Arabes mentent, il n'en est pas moins certain qu'ils sont très hostiles à l'égard des Espagnols, ce qui laisse supposer que Cide Hamete Benengeli a probablement volontairement minimisé les exploits de Don Quichotte. Le lecteur doit donc conclure qu'en réalité ses actes héroïques ont certainement dû être encore beaucoup plus grandioses que ce qu'en dit le narrateur arabe ${ }^{5}$. Évidemment, le passage regorge d'ironie, non seulement à l'égard du héros et du narrateur, mais surtout à l'égard du vieux préjugé que les poètes sont des menteurs. Apparemment, déjà le premier roman moderne pouvait se permettre de jouer de manière ironique avec le reproche de mensonge. Mais si, même pour la réception traditionnelle et "normale" de la littérature, il n'est plus question de mensonge, on peut se demander dans quelle mesure il pourrait être légitime de parler quand même de «mensonge littéraire »? Car si la littérature ne savait mentir, on pourrait soupçonner que lorsqu' on parle de « mensonge littéraire », il s'agit souvent d'une métonymie ou d'une simple métaphore. Dans la plupart des publications sur le mensonge dans la littérature, celui-ci intervient seulement à titre d'objet de la représentation littéraire. Ainsi l'on peut évidemment parler, d'une manière métonymique, de mensonge romanesque pour évoquer des personnages menteurs dans La verdad sospechosa d'Alarcón ${ }^{6}$, Le Menteur de Corneille ${ }^{7}$ ou bien Le Tartuffe de Molière ${ }^{8}$. Le même argument vaut pour la métaphore, car souvent on emploie l'expression «mensonge littéraire » de manière figurée, ne conservant en cela qu'un seul trait du mensonge, comme par exemple la divergence entre opinion et énonciation qui est valable pour presque toutes les figures de style et tous les tropes, sans tenir compte de la deuxième condition, à savoir la dissimulation de

4 Miguel de Cervantes SaAvedra, El ingenioso hidalgo Don Quijote de la Mancha. éd. par Francisco Rico, Madrid, Galaxia Gutenberg, 2005, p. 120.

5 Ibid.

6 Juan Ruiz de Alarcón, La verdad sospechosa, Madrid, Cátedra, 1990.

Pierre Corneille, Le Menteur, éd par Geneviève Winter, Paris, Gallimard, 2006.

Molière, Le Tartuffe ou L'Imposteur, éd. par Françoise Rullier-Theuret, Paris, Larousse, 2011. 
cette divergence. En fait, quand on utilise des tropes littéraires tels que « l'oiseau de la liberté », il y a certes une divergence entre conviction et expression, mais cette divergence n'est point celée. Elle est, tout au contraire, signalée ouvertement par le code littéraire même. Si l'on parle donc, dans ce cas, de « mensonge littéraire », cela se fait évidemment dans un sens métaphorique.

Tous les travaux sur le mensonge littéraire, qui analysent surtout la représentation du mensonge et du menteur dans le roman et dans le théâtre, enrichissent incontestablement notre connaissance du mensonge et de son fonctionnement ${ }^{9}$. Cependant, une telle approche connaît malgré tout des limites, car elle se borne à appliquer une certaine notion du mensonge à l'histoire racontée pour examiner de quelle manière il y est représenté. Un défi théorique plus important consisterait à se demander si, dans le cadre établi ci-dessus, nous pouvons concevoir l'idée d'un mensonge qui serait spécifique à la littérature ou à l'esthétique en général, c'est-à-dire une manière de mentir caractéristique de la littérature et de ses codes. Il y aurait donc deux manières bien distinctes d'aborder la relation entre littérature et mensonge: soit appliquer une définition et une théorie du mensonge développées par d'autres disciplines que la littérature, pour examiner dans quelle mesure l'esthétique en général et la littérature en particulier sont susceptibles de mentir; soit renverser la relation entre théorie générale du mensonge et théorie littéraire, et se demander sous quel jour le mensonge apparaît à la lumière de la littérature, ou bien ce que la théorie littéraire peut apporter à une théorie générale du mensonge.

\section{Formes du mensonge en littérature}

Dans un roman, le mensonge peut se situer à plusieurs endroits. En effet, la narratologie distingue plusieurs niveaux de la communication littéraire, dont le plus visible est évidemment celui de la communication interne entre les personnages. Ici, nous sommes confrontés aux menteurs et aux mensonges au niveau de l'énoncé littéraire, c'est-à-dire sur le plan de la macrostructure, à savoir la constellation des personnages et l'histoire ${ }^{10}$. Depuis ses débuts jusqu'à « l'extrême contemporain », la littérature a toujours représenté des menteurs et des mensonges. Il suffit d'évoquer les plus mémorables: La Bible avec Adam et Ève, le Serpent, Jacob, saint Pierre, l'Odyssée avec Ulysse, le Iago dans Othello de Shakespeare, le picaro de Lazarillo de Tormes, le Don García de La verdad sospechosa d'Alarcón, le Dorante du Menteur de Corneille, le Tartuffe ou le Dom Juan de Molière, Claggart dans Billy Budd de Melville, Pinocchio de Collodi, dont le nom est devenu le symbole du mensonge, le Stavrogin dans Les Démons de Dostoievski, le Felix Krull de Thomas Mann,

9 À titre d'exemples: Gilles Barbedette, L'Invitation au mensonge. Essai sur le roman, Paris, Gallimard, 1989; Anne-Marie Baron, Balzac ou L'Auguste Mensonge, Paris, Nathan 1998; Llewellyn Brown, Figures du mensonge littéraire. Études sur l'écriture au XXe siècle, Paris, L'Harmattan, 2005 ; Maria Bettetini, Eine kurze Geschichte der Lüge. Von Odysseus bis Pinocchio, Berlin, Wagenbach, 2003.

${ }^{10}$ Rolf Fieguth, « Zur Rezeptionslenkung bei narrativen und dramatischen Werken », Sprache im technischen Zeitalter, $\mathrm{n}^{\circ}$ 43, 1973, p. 186-201. 
Tom Buchanan dans The Great Gatsby de Scott Fitzgerald, l'Albertine de $\grave{A}$ la recherche du temps perdu de Marcel Proust, le Thomas de Fontenoy de Thomas l'imposteur de Jean Cocteau ou bien le tricheur des Mémoires d'un tricheur de Sacha Guitry. Dans toutes ces œuvres, les menteurs et le mensonge sont des thèmes centraux. Néanmoins, dans tous ces exemples, il ne s'agit évidemment point d'une forme spécifiquement littéraire ou esthétique du mensonge.

Cependant, nous trouvons le mensonge littéraire non seulement au niveau de l'histoire, mais aussi au niveau de sa narration. Dans ce cas-là, le narrateur d'une histoire fait la même chose que le héros de l'histoire: il induit sciemment quelqu'un en erreur, le cas échéant le lecteur. Ce passage du mensonge de l'histoire à la narration se fait d'une manière plus continue et moins perceptible si le héros est en même temps le narrateur de son histoire. Ainsi, dans La Symphonie pastorale, le pasteur relate dans son journal intime comment il accueille dans sa famille une jeune orpheline aveugle qu'il se propose d'éduquer et dont il veut élever l'âme. Or, le narrateur passe sous silence sa passion grandissante pour Gertrude, à tel point qu'il essaie d'empêcher son fils Jacques de l'épouser ${ }^{11}$. Le récit autodiégétique attribue toujours les actions du pasteur aux motifs les plus nobles et les plus altruistes, jusqu'à ce que la vérité brutale éclate: le pasteur a été la victime des désirs que Gertrude lui a inspirés. Gide conçoit donc un cas où non seulement le héros ment à ses enfants et à sa famille, mais où il ment également à son lecteur. Toutefois, dans le cas de La Symphonie pastorale, le statut du mensonge est équivoque, car il appartient en même temps à l'histoire et au discours narratif. Comme le roman prend la forme d'un journal intime, on ne peut pas décider si le pasteur se ment simplement à luimême, ce qui relèverait de l'histoire racontée, ou bien si le narrateur ment également au lecteur, ce qui relèverait du discours narratif. Le roman de Gide puise une partie de son intérêt esthétique dans cette ambiguïté fondamentale.

Le statut du mensonge est plus clair quand le narrateur homodiégétique est simplement le témoin des événements racontés. On peut illustrer ce type de mensonge littéraire situé entièrement au niveau du discours narratif à l'aide d'un texte devenu célèbre dans l'histoire littéraire : le roman policier The Murder of Roger Ackroyd d'Agatha Christie ${ }^{12}$. L'histoire relate comment le détective Hercule Poirot essaie d'élucider, comme le titre l'indique, l'assassinat de Roger Ackroyd. Selon le modèle créé par Conan Doyle, les événements sont racontés par le docteur James Sheppard, un témoin qui joue de temps à autre le rôle d'assistant du détective. Le narrateur ne dispose que d'un savoir limité sur l'histoire du crime et sur les pensées du détective dont les actions restent souvent énigmatiques pour lui, ce qui se traduit sur le plan narratif par une focalisation interne sur Sheppard et qui correspond à une focalisation externe sur l'assassin inconnu et Hercule Poirot. Jusqu'au bout du récit, le docteur Sheppard semble ne pas deviner qui est soupçonné par Hercule Poirot et encore moins qui est l'assassin. Or, à la fin de l'histoire, il se révèle que le docteur a été un narrateur non fiable qui a dissimulé des événements au lecteur et en a rajouté d'autres. Il s'avère qu'il est lui-même l'assassin. Cependant, il y a une différence

\footnotetext{
11 André Gide, La Symphonie pastorale, Paris, Gallimard, 1998.

12 Agatha Christie, The Murder of Roger Ackroyd, London, Harper Collins, 2002.
} 
considérable entre un narrateur non fiable classique et le docteur Sheppard d'Agatha Christie. En général, selon la définition classique de Wayne Booth, un narrateur non fiable se définit par sa déviance ou bien sa discordance avec les normes de l'auteur implicite ${ }^{13}$. Si l'on préfère une formule moins personnalisée, on peut dire également que le narrateur non fiable s'écarte des normes établies par l'œuvre littéraire ellemême. Toujours est-il que cette déviance ou discordance doit être signalée par l'œuvre elle-même, sinon elle serait complètement inopérante. Un narrateur non fiable, si l'expression fait sens, doit être signalé comme tel par l'auteur, sinon il ne joue aucun rôle lors du processus de lecture. Or, dans la perspective de la théorie du mensonge, la narration non fiable ne constitue pas un mensonge, car le mensonge potentiel est signalé par l'œuvre elle-même. Ainsi, le narrateur non fiable ne représente, sous forme de mise en abyme, qu'un cas particulier du « mensonge littéraire » en général. Tout comme celui-ci est signalé par les signes de la fictionnalité, la non fiabilité du narrateur est signalée par la discordance entre les opinions du narrateur et le monde du roman lui-même. Or, dans le roman d'Agatha Christie, nous ne rencontrons de la part du docteur Sheppard aucun signe de déviance par rapport au monde romanesque qui pourrait mettre en garde le lecteur contre un trucage éventuel. Le docteur est entièrement conforme au monde du roman, ainsi qu'aux normes de l'auteur implicite. C'est à la toute fin du roman qu'il apparaît comme l'assassin. Nous sommes donc confrontés à un véritable mensonge du narrateur, car il nous dissimule ce qu'il sait et simule n'être que le simple investigateur d'un crime dont il est en réalité l'auteur. Comme nous l'avons vu, il n'y a aucun signe apparent de mensonge à la première lecture du roman. Nous sortons donc du système littéraire de la narration non fiable. Ce n'est qu'à la deuxième lecture que le lecteur peut voir à quel point il est passé à côté de certains indices qui désignaient Sheppard comme l'auteur du crime. En fait, le mensonge du docteur peut passer parfaitement inaperçu parce qu'en même temps qu'il ment au niveau de l'histoire et de la narration, il contrevient aux normes du roman policier.

Ce serait donc également Agatha Christie, ou bien l'auteur implicite, qui serait à l'origine d'un véritable mensonge spécifiquement littéraire: ce dernier se réalise grâce au pacte littéraire lui-même, dont elle feint de respecter les règles implicites pour induire son lecteur en erreur. Selon ces règles implicites du roman policier, l'histoire se divise en général en deux histoires, la première correspondant à celle du crime et la deuxième à celle de son élucidation ${ }^{14}$. Chaque histoire comporte une constellation de personnages différente, à savoir celle du crime constituée principalement de l'assassin et des victimes, et celle de l'investigation se composant du détective et des suspects. Or, à la différence du thriller, la règle du roman policier classique présuppose une séparation nette entre l'histoire du crime et celle de son investigation ${ }^{15}$. De ce fait, le narrateur se trouve en général du côté du détective et ne fait point partie des suspects. C'est pour cette raison que la plupart des lecteurs

13 Wayne C. Воотн, The Rhetoric of Fiction, Chicago, University of Chicago Press, 1961, p. 158 sq.

${ }^{14}$ Cf. Tzvetan Todorov, « Typologie du roman policier », in Poétique de la prose, Paris, Seuil, 1978, p. 919, ici p. 11.

15 Ibid., p. 14. 
passent à côté des indices qui signalent le docteur Sheppard comme l'assassin, car ces indices-là contreviennent aux conventions littéraires. Ainsi, le mensonge se trouve aussi à un niveau narratif supérieur, car ce n'est pas seulement le narrateur qui ment, mais aussi l'auteur implicite qui abuse le lecteur implicite, en adoptant en apparence les règles du roman policier qu'il a déjà abandonnées en secret. Toutes les conditions pour un mensonge seraient donc remplies: primo, une divergence entre opinion (infraction aux codes du roman policier) et expression (respect apparent de ces mêmes codes), secundo la dissimulation de cette divergence et tertio des objectifs supérieurs (ici un effet esthétique de surprise pour le lecteur et peut-être aussi une prise de conscience des règles du roman policier).

Évidemment, le roman policier classique constitue un cas facilement analysable de mensonge littéraire, car il s'agit là d'un genre hautement codifié. Mais nous trouvons également des exemples dans des genres qui le sont moins. Dans le roman Je m'en vais de Jean Echenoz, conduit en focalisation zéro, un narrateur apparemment omniscient raconte l'histoire du galeriste Ferrer qui, après la mort soudaine de son assistant Delahaye, entreprend une expédition au pôle Nord au cours de laquelle il trouve un trésor d'œuvres d'art. Mais malheureusement, ces œuvres lui sont volées après son retour en France. Dans la suite de l'histoire, le roman raconte en narration alternée les méfaits de l'auteur du vol, le gangster Baumgartner, et les tentatives de Ferrer pour récupérer les œuvres perdues. À la fin du roman, Ferrer réussit à coincer Baumgartner. Mais au grand étonnement du lecteur, Ferrer découvre que ce Baumgartner n'est personne d'autre que Delahaye: «Tiens, dit Ferrer, Delahaye. Je me disais bien, aussi ${ }^{16} . »$ Mais ce qui est une véritable découverte pour Ferrer et le lecteur ne devrait pas l'être pour le narrateur omniscient. Celui-ci a donc dissimulé au lecteur la véritable identité de Baumgartner, qu'il est censé connaître parfaitement bien. Dans ce cas spécial, nous trouvons également réunies toutes les conditions du mensonge: 1 . Une divergence entre opinion et expression, car l'auteur sait que Delahaye et Baumgartner ne font qu'un et fait croire au lecteur qu'il s'agit de deux personnages différents. 2. La dissimulation de cette divergence entre ce qu'il sait et ce qu'il dit. 3. Son utilisation pour réaliser des objectifs esthétiques, comme par exemple provoquer la surprise du lecteur.

À l'opposé des romanciers modernes qui dénoncent les conventions romanesques pour rompre avec elles, Jean Echenoz fait semblant de les respecter, alors qu'il se borne à s'en servir pour tromper le lecteur. Tandis que la pratique singulière d'Echenoz présuppose la validité incontestée du code littéraire dont il use pour abuser le lecteur, et qu'elle constitue donc de ce fait un véritable exemple de mensonge romanesque et d'inauthenticité, le roman moderne - en particulier le Nouveau Roman - dénonce l'inauthenticité des conventions romanesques mensongères. Le texte d'Echenoz se démarque de cette logique moderne de la surenchère, du dépassement et de la destruction. Il ne dépasse point la forme conventionnelle mais triche avec elle, ment grâce à elle, tout en restant volontiers en deçà des normes romanesques et en constituant une espèce de " sous-enchère » esthétique.

16 Jean Echenoz, Je m'en vais, Paris, Minuit, 1999, p. 227. 
Jusqu'ici nous avons établi différents types de mensonge narratif: au niveau de l'histoire, de la narration, et de la composition par l'auteur implicite. Mais il existe une autre forme de mensonge spécifiquement littéraire qui se situe à un niveau narratif encore supérieur et qui concerne cette fois-ci la communication entre l'auteur comme producteur du texte et le lecteur comme destinataire littéraire. À ce niveau-là, le pacte littéraire ou le pacte fictionnel lui-même est concerné. Si celui-ci consiste en un code de la fiction qui signale lui-même que le texte que nous lisons est purement inventé et qu'il dénonce donc le mensonge tout en le supprimant, on peut se demander s'il existe des œuvres littéraires qui violent ce pacte. Ce serait le cas si un auteur mélangeait pacte fictionnel et pacte référentiel: les signes de la fictionnalité ne fonctionneraient plus comme des signaux indiquant la divergence entre conviction et opinion d'une part, et expression ou énonciation d'autre part. Cette divergence resterait dissimulée comme dans un mensonge normal. Une œuvre qui joue à la fois sur le registre de la fiction et sur celui d'un discours référentiel livre un exemple de ce mécanisme, présent dans tous les genres hybrides tels que la docufiction ou l'autofiction. Dans Soldados de Salamina de Javier Cercas ${ }^{17}$, héros et narrateur autobiographique portent le même nom que l'auteur, ce qui fait croire au lecteur qu'il a affaire à un " pacte autobiographique », où auteur, narrateur et héros sont identiques ${ }^{18}$. D'autres éléments du texte, tels des personnages célèbres de la guerre civile espagnole, des lieux, des événements historiques et des auteurs connus, comme Roberto Bolaño, suggèrent également qu'il s'agit d'un texte référentiel. Mais si le lecteur peut authentifier certains faits de la biographie du Javier Cercas du livre, il est amené à constater qu'il y en a d'autres qui sont complètement inventés. Le lecteur est donc mis sur une fausse piste. Ce qui se passe ici est clair: c'est grâce au code des genres que l'auteur est parvenu à tromper le lecteur. Les mêmes « mensonges littéraires » sont à l'œuvre chaque fois que nous sommes confrontés à un texte dont le statut factuel ou fictionnel est équivoque. En fait, ce qui dérange dans des textes comme Fils ou Le Livre brisé de Serge Doubrovsky, dans les docufictions de François Bon ou bien dans la nouvelle autobiographie d'Alain Robbe-Grillet, c'est qu'ils nous laissent croire qu'il s'agit là de textes référentiels relevant $\mathrm{du}$ «pacte autobiographique » ou de textes référentiels dotés d'instances narratives bien ancrées dans la réalité, faisant intervenir des lieux et des personnages du monde réel, mais qu'ils y insèrent subrepticement des épisodes inventés, ce qui confère à l'auteur un statut équivoque qui oscille entre factualité et fictionnalité.

Nous pouvons donc conclure que même s'il n'y a pas de mensonge littéraire primaire, ou au sens propre du terme, il y a néanmoins des mensonges littéraires secondaires, au sein même du discours littéraire. Ce type de mensonge consiste en une divergence entre, d'une part, ce qui peut être considéré comme la position ou la tendance générale de l'œuvre, et d'autre part ses manifestations esthétiques concrètes, entre prétention et réalisation esthétique. Dans une conférence intitulée La verdad de las mentiras, Mario Vargas Llosa, tout en reconnaissant l'incapacité de la littérature à mentir au sens propre du terme, parle toutefois de l'existence d'un

\footnotetext{
17 Javier Cercas, Soldados de Salamina. Barcelona, Tusquets, 2007.

18 Philippe Lejeune, Le Pacte autobiographique, Paris, Seuil, 1996.
} 
mensonge littéraire ${ }^{19}$. Pour lui, la fiction elle-même, son monde, ses personnages peuvent être considérés comme la «vérité inhérente» au texte ou bien comme « la conviction » de l'auteur implicite ou du narrateur. Cette tendance générale de l'œuvre correspond, sur un certain plan, à ce que W. Booth appelle les normes de l'auteur implicite. Si nous considérons cela comme la conviction ou bien l'opinion de l'œuvre, le texte lui-même peut bien sûr converger vers cette tendance générale ou au contraire s'en écarter. Chez Booth, le premier cas était nommé narration fiable, le second narration non fiable. Si l'auteur réussit à trouver une forme adéquate pour la représentation de ce monde fictionnel, s'il y a donc une correspondance entre le monde fictif d'une part et son expression esthétique d'autre part, l'œuvre serait authentique. Mais si cela n'est pas le cas, si le texte tente en même temps de dissimuler cette divergence, ce qui se produit dans la plupart des textes littéraires qui manquent de cohérence interne, nous avons affaire - selon Vargas Llosa - à un mensonge littéraire ${ }^{20}$. L'objectif de ce type de mensonge littéraire consiste à tromper le lecteur sur la qualité esthétique du texte. Les romans kitsch, comme le texte suivant de Delly, une auteure de littérature populaire à succès de la première moitié du $\mathrm{Xx}^{\mathrm{e}}$ siècle, en offrent un exemple:

\footnotetext{
De tous les hommes qui étaient là, aucun ne pouvait se vanter d'égaler quelque peu l'être d'harmonieuse beauté et de suprême élégance qu'était Élie de Ghiliac. Ce visage aux lignes superbes et viriles, au teint légèrement mat, à la bouche fine et railleuse, cette chevelure brune aux larges boucles naturelles, ces yeux d'un bleu sombre, dont la beauté était aussi célèbre que les œuvres de M. de Ghiliac, et la haute taille svelte, et tout cet ensemble de grâce souple, de courtoisie hautaine, de distinction patricienne faisaient de cet homme de trente ans un être d'incomparable séduction ${ }^{21}$.
}

Il est évident que l'auteure a voulu créer un personnage extraordinaire qui évolue dans un monde non moins extraordinaire. Il apparaît cependant clairement que la forme que Delly donne à ce monde fictif produit un effet contraire. La surcharge d'épithètes extraordinaires rend l'extraordinaire ordinaire et l'usage exagéré du superlatif subvertit la crédibilité du texte et produit un effet de distanciation involontaire. S'y ajoute une histoire dont les péripéties et la fin - bien sûr heureuse - sont prévisibles dès les premières lignes. La tendance du texte à créer des personnages et une histoire hors du commun se trouve donc contredite dans sa forme commune et triviale. Le texte se sert de stéréotypes et de clichés au lieu de développer une description particulière d'un personnage singulier, tout en essayant également de cacher cet écart, dans le dessein de conserver l'intérêt du lecteur. Toutes les conditions du mensonge sont donc remplies, mais comme ce mensonge relève du monde de la fiction ou bien de la communication strictement littéraire, il s'agit d'un mensonge secondaire, qui fonctionne à l'intérieur de la véracité de base du pacte fictionnel. La référence de cette critique est évidemment constituée par la

19 Mario Vargas Llosa, La Verdad de las mentiras. Ensayos sobre literatura, Barcelona, Seix Barral, 1990, p. 5-20.

${ }^{20}$ Ibid., p. 10.

${ }^{21}$ Delly, Entre deux âmes, Montréal, La Bibliothèque électronique du Québec (collection «Classiques du XXe siècle»), vol. 99 [http://beq.ebooksgratuits.com/classiques/Delly-ames.pdf; $1^{\text {er }}$ mars 2014], p. 6 sq. 
notion « magique » d'authenticité - comme la qualifiait jadis Theodor W. Adorno -, véritable impératif catégorique de l'esthétique moderne, qui incite tout auteur à se méfier de l'imitation des poncifs et l'oblige à trouver son style individuel, sa forme littéraire originale ${ }^{22}$. Si la modernité peut se définir comme ce mouvement permanent de destruction des formes traditionnelles et de leur remplacement par des formes nouvelles, si elle se caractérise par la recherche de moyens pour exprimer de manière authentique le moment présent que Charles Baudelaire a si bien décrite dans Le Peintre de la vie moderne, la littérature est susceptible de mentir. Car si un texte littéraire est incapable de correspondre par sa forme littéraire à la "réalité" ou la "vérité" fictive et donc "secondaire" du monde romanesque créé par lui, il constitue un véritable mensonge esthétique. Ainsi, dans une conférence célèbre, Hermann Broch a déclaré que le kitsch était la forme littéraire du mensonge. Comme le mal dans la religion, le kitsch est identifié au mal dans le système de valeurs de l'art ${ }^{23}$. Dans le cas de Delly, le mensonge esthétique naît d'une divergence entre la prétention du monde fictif d'une part, et l'incapacité de l'auteure à créer une forme narrative qui lui corresponde d'autre part. Mais nous pouvons également concevoir un mensonge littéraire où la forme générale du roman conventionnel empêcherait l'auteur de trouver une expression adéquate de ce qu'il pense ou ce qu'il sent. Avec ce phénomène, nous sommes confrontés à toute une tradition de critique du langage qui commence avec l'essai de Nietzsche Über Wahrheit und Lüge im außermoralischen $\operatorname{Sinn}^{24}$, en passant par le célèbre Ein Brief (dit Chandos-Brief) de Hugo von Hofmannsthal ${ }^{25}$, pour culminer ensuite dans le reproche formulé par Roland Barthes lors de sa Leçon inaugurale au Collège de France, où il affirme que le langage est « tout simplement fasciste ${ }^{26}$ ». Mais en dehors de cette critique radicale du Barthes tardif, le jeune Barthes des années cinquante a développé, dans Le Degré zéro de l'écriture, une critique du système romanesque qui revient également à considérer le roman conventionnel comme un mensonge esthétique. Selon Barthes, le passé simple, le récit à la première personne et la structure téléologique du roman obligent le romancier à s'exprimer au moyen d'une forme figée, fausse et mensongère : «On s'explique alors ce que le passé simple du Roman a d'utile et d'intolérable: il est un mensonge manifesté ${ }^{27} \gg$. Le roman de facture conventionnelle contraindrait donc le

${ }^{22}$ Cf. Jochen Mecke, « Der Prozess der Authentizität », in Susanne KnAller, Harro Müller (dir.), Authentizität, Paderborn, Fink, 2006, p. 82-114.

${ }^{23}$ «Der Kitsch ist das Böse im Wertesystem der Kunst» (Hermann BRoch, «Zum Problem des Kitsches ", in Hermann Broch, Die Idee ist ewig. Essays und Briefe, München, dtv, 1968, p. 117-132, ici p. 128).

${ }^{24}$ Friedrich Nietzsche, « Über Wahrheit und Lüge im außermoralischen Sinn », in Werke, vol. III, éd. par Karl SchlechTA, Frankfurt, Ullstein, 1972, p. 1017-1030.

${ }^{25}$ Hugo von Hofmannsthal, «Ein Brief» (1902), in Gesammelte Werke in zehn Einzelbänden, vol. VII, Erzählungen. Erfundene Gespräche und Briefe. Reisen, Frankfurt a. M., Fischer, 1979, p. 461-472.

26 « La langue, comme performance de tout langage, n'est ni réactionnaire ni progressiste; elle est tout simplement fasciste; car le fascisme, ce n'est pas d'empêcher de dire, c'est d'obliger à dire. » (Roland BARTHES, Leçon: leçon inaugurale de la chaire de sémiologie littéraire du Collège de France, prononcée le 7 janvier 1977, Paris, Seuil, 1978, p. 14).

${ }^{27}$ Roland BARTHES, «Le degré zéro de l'écriture », in Le Degré zéro de l'écriture, suivi de Nouveaux essais critiques, Paris, Seuil, 1972, p. 7-65, ici p. 27. 
romancier à produire un mensonge qui trahirait ses propres convictions et intentions esthétiques. L'écriture romanesque est conçue comme une espèce de fatalité littéraire qui mène directement au mensonge esthétique, et ce indépendamment de la volonté du romancier individuel. Ainsi Barthes développe, dans le domaine esthétique, une notion que Walter Benjamin avait conçue pour le domaine sociopolitique, c'est-àdire l'idée d'un « mensonge objectif » qui est le produit d'une contrainte structurelle, indépendamment de toute volonté individuelle de mentir ${ }^{28}$.

\section{Pour une théorie littéraire du mensonge}

Dans ce qui précède, nous avons envisagé la littérature à l'aune d'une notion et d'une théorie générales du mensonge, pour nous demander s'il existe un mensonge spécifiquement littéraire. Mais on peut inverser l'ordre du questionnement et examiner sous quel jour le mensonge apparaît à la lumière de la littérature, et si la littérature et l'esthétique peuvent apporter quelque chose à une théorie générale $d u$ mensonge. Si nous reprenons les termes de notre définition, à savoir qu'un mensonge consiste en une divergence entre ce que nous pensons ou bien ressentons d'une part, et l'expression que nous essayons de lui donner d'autre part, il apparaît tout de suite que le mensonge relève d'une problématique de l'expression et de la forme, et comporte donc une dimension esthétique.

Tout d'abord, la littérature pourrait aider à mieux comprendre le problème de la reconnaissance du mensonge. Le symbole littéraire le plus visible de cette problématique est évidemment Pinocchio. Mais en réalité, le personnage littéraire qui incarne peutêtre le mieux le mensonge, à tel point qu'il en est devenu l'icône, ment très peu et s'il le fait, c'est toujours pour se protéger lui-même ou protéger ses amis quand il y a un danger ${ }^{29}$. Pinocchio a quand même pu devenir le symbole du mensonge parce qu'il semble compenser une blessure narcissique qui, en plus des blessures copernicienne, darwinienne et freudienne ${ }^{30}$, constitue une blessure sémiotique. Comme l'acte de mentir consiste en une dissimulation de la divergence entre pensée et expression ou signe, il inflige une véritable humiliation à l'homme qui n'est plus maître des signes, car il ne peut reconnaître le mensonge comme tel. Il en naît un véritable « complexe de Pinocchio », dont le héros de Collodi, les machines à détecter le mensonge et des séries américaines comme Lie to me ne sont que les manifestations les plus connues ${ }^{31}$. Dans ce contexte, la littérature elle-même peut être considérée comme une compensation permanente de cette blessure narcissique, car elle permet de réaliser le rêve de pouvoir reconnaître tout mensonge. Tandis que dans la vie quotidienne nous n'avons pas la possibilité de connaître les véritables pensées d'une personne, la littérature, quant à elle, nous apprend bien les pensées intimes d'autrui et nous rend de ce fait capable de

28 Walter Benjamin, « Notizen über "Objektive Verlogenheit” I », in Walter BENJAmin, Gesammelte Schriften, t. VI, éd. par Rolf Tiedemann et Hermann SchweppenhäUser, Frankfurt a. M., Suhrkamp, 1985, p. 60-62.

${ }^{29}$ Carlo Collodi, Pinocchios Abenteuer. Die Geschichte einer Holzpuppe, Stuttgart, Reclam, 2008.

30 Sigmund Freud, Introduction à la psychanalyse, Paris, Payot, 2001, p. 33.

31 Brian Grazer, David Nevins, Samuel Baum, Lie to me, USA, Fox, 2009-2011. 
savoir s'il ment. En fait, la littérature a développé de multiples techniques pour codifier le mensonge tout en révélant la divergence entre conviction/ sentiment d'une part et expression/ énonciation d'autre part, une divergence qui peut s'établir à plusieurs niveaux narratifs, c'est-à-dire au niveau des personnages, mais aussi au niveau du narrateur et de l'auteur, ainsi que de l'œuvre.

Pour ce qui est du premier niveau, la focalisation interne et le discours indirect libre ont évidemment constitué un pas décisif dans la représentation du mensonge, car ils ont permis de créer l'impression d'avoir accès à la conscience d'un personnage, de pouvoir prendre connaissance de ses pensées les plus intimes et de disposer d'une échelle à laquelle on puisse mesurer le mensonge. La fascination de la littérature réside en ceci qu'elle permet de connaître les pensées cachées ou dissimulées d'autrui et de fonctionner de cette manière comme un véritable "détecteur de mensonge" qui compense la blessure narcissique sémiotique due au mensonge.

Si la conception courante du mensonge a tendance à dissimuler sa dimension esthétique, c'est en général parce qu'elle part de l'idée que les formes linguistiques ou sémiotiques destinées à exprimer nos pensées sont toujours à notre disposition. Selon cette idée répandue, nos énoncés sont toujours contrôlés par notre intention, qui, comme Jacques Derrida l'a souligné dans Histoire du mensonge. Prolégomènes, est considérée comme une présence-à-soi ${ }^{32}$. Mais c'est justement ici que la prise en considération de la dimension esthétique subvertit la présupposition cachée de la théorie conventionnelle du mensonge selon laquelle l'expression est une espèce de forme transparente qui se moule parfaitement sur le contenu. Elle permet de concevoir l'idée que nous pouvons mentir "malgré nous" parce que la langue, c'est-à-dire la forme, nous a fourché et que nous n'arrivons pas à dire ce que nous voulons dire. Si nous imaginons un acte sémiotique qui, au lieu de nous empêcher de dire ce que nous voulons, nous fait dire autre chose que ce que nous voulons, nous nous approchons du mensonge. Nous nous servons alors d'un langage qui trahit notre pensée au lieu de l'exprimer. C'est le cas si les expressions que nous choisissons sont marquées par l'empreinte d'une idéologie opposée à notre conviction personnelle. Si par exemple quelqu'un veut exprimer l'idée que l'homme peut s'émanciper de la nature et des contraintes sociales grâce au travail, et s'il utilise, en allemand, les paroles « Arbeit macht frei », il exprime certainement autre chose que ce qu'il pense, car il reprend, peut-être même à son insu, le slogan cynique que les nazis avaient inscrit à l'entrée des camps de concentration. Ainsi, la pensée initiale a été détournée par l'expression choisie, et si ce détournement reste dissimulé, la véracité subjective s'est transformée en mensonge objectif. Mais nous n'avons point besoin de recourir à des exemples aussi spectaculaires. À ce titre, Madame Bovary est très instructif:

Eh quoi! dit-il [i.e. Rodolphe], ne savez-vous pas qu'il y a des âmes sans cesse tourmentées? Il leur faut tour à tour le rêve et l'action, les passions les plus pures, les jouissances les plus furieuses, et l'on se jette ainsi dans toutes sortes de fantaisies, de folies. Alors elle le regarda comme on contemple un voyageur qui a passé par des pays extraordinaires, et elle reprit: - Nous n'avons pas même cette distraction, nous autres pauvres femmes! - Triste distraction, car on n'y trouve pas le bonheur ${ }^{33}$.

\footnotetext{
32 Jacques Derrida, Histoire du mensonge. Prolégomènes, Paris, l'Herne, 2005, p. 24.

33 Gustave Flaubert, Euvres complètes, t. I, Madame Bovary, Paris, Lévy, 1857, p. 204.
} 
Ici, Rodolphe se sert évidemment de manière délibérée et mensongère des topiques du romantisme qui se sont transformés en des formules toutes faites. Mais contrairement à Rodolphe, pour Emma, ces clichés traduisent ses espoirs et ses désirs les plus intimes, alors que leur caractère usé et stéréotypé lui reste caché. Cela apparaît clairement lors des trois jours de «lune de miel » passés avec son amant Léon à Rouen, où ils se laissent aller à leurs penchants romantiques :

\section{Une fois, la lune parut; alors ils ne manquèrent pas à faire des phrases, trouvant l'astre mélancolique et plein de poésie; même elle se mit à chanter: Un soir, t'en souvient-il? nous voguions, etc. ${ }^{34}$}

Ici, la divergence ne s'ouvre plus, comme dans le cas de Rodolphe, entre conviction et expression, mais entre les sentiments d'Emma et de Léon d'une part, et les moyens linguistiques qu'ils ont à leur disposition pour les exprimer d'autre part. Et il semble qu'il en soit de même pour le narrateur dont les formules comme « faire des phrases » et « etc. » ne sont certainement pas aptes à exprimer l'intensité des sentiments des deux amoureux ${ }^{35}$. Si nous considérons les sentiments des deux protagonistes, leur monde et leur conscience comme la "vérité" interne du passage cité, il s'agit d'un mensonge, car l'expression n'est point capable de leur correspondre. Mais ce mensonge est d'ordre esthétique, car la divergence entre conviction et expression s'installe malgré la bonne foi apparente des protagonistes. Or, cette incapacité ostensible des personnages remplit une fonction bien précise dans l'esthétique de Flaubert. Car les expressions plates et banales correspondent à quelque chose de plus profond que la banalité des émotions et des sentiments d'Emma et de Léon: elles renvoient à une inauthenticité de fond. Ce qu'elles dénoncent, c'est le caractère stéréotypé du discours romantique qui sert de modèle à Emma et Léon, mais qui, en réalité, met à leur disposition un langage de seconde main, où ils ne trouvent que des formules toutes faites, des stéréotypes et des clichés.

Le texte de Flaubert nous révèle que le mensonge comporte une problématique de l'expression et du langage, et nous amène à concevoir l'idée d'un mensonge non intentionnel, dû au fait que le dire ne correspond pas au vouloir-dire, ou imputable à notre incapacité à exprimer ce que nous ressentons. Mais à la différence d'une incapacité individuelle, celle-ci est pour ainsi dire "institutionnalisée". En fait, si Emma pense de Charles que sa « conversation [...] était plate comme un trottoir de rue, et [que] les idées de tout le monde y défilaient dans leur costume ordinaire ${ }^{36}$ ", cela vaut aussi pour elle-même, même si ses idées à elle s'opposent à celles de son mari. Nous avons donc affaire à un type de mensonge qui ne relève plus de l'éthique ou de la morale: il ne dépend plus du sujet de l'énonciation mais constitue une espèce de mensonge esthétique «objectif», qui partage quelques traits caractéristiques avec le type de mensonge dont parle W. Benjamin dans l'esquisse citée plus haut ${ }^{37}$.

\footnotetext{
${ }^{34}$ Ibid., p. 362.
}

35 Contrairement à ses personnages, Flaubert est évidemment parfaitement conscient de ce clivage entre sentiments et expressions, et il s'en sert dans le passage cité pour créer un effet de distanciation ironique.

${ }^{36}$ Flaubert, Madame Bovary, p. 59.

37 Cf. n. 28. 
Benjamin conçoit l'idée d'un mensonge qui ne relèverait plus de l'individu, mais qui serait plus profond que lui et ancré dans la collectivité d'une société, d'une nation ou d'une culture. Flaubert, dans Madame Bovary et L'Éducation sentimentale, en montre la dimension esthétique, qui ressortit non seulement à une problématique de l'expression, mais aussi à la sensibilité, à la perception et à la sensation, comme l'indique l'étymologie de aisthesis. C'est à cet endroit précis, et non pas comme une théorie générale ou une philosophie du langage, que l'on peut situer la pertinence de la conception du langage développée par Nietzsche dans Über Wahrheit und Lüge im außermoralischen Sinn et de celle exposée par Barthes dans sa Leçon inaugurale au Collège de France. Les deux théories, dont il a été question plus haut, trouvent en Flaubert leur prédécesseur.

Cependant, Flaubert pousse ses analyses encore plus loin, car il sonde également la conscience subjective. Dans le chapitre consacré à l'éducation d'Emma, il détecte les sources de ses attitudes dans une littérature schématique truffée de clichés:

Ce n'étaient qu'amours, amants, amantes, dames persécutées s'évanouissant dans des pavillons solitaires, postillons qu'on tue à tous les relais, chevaux qu'on crève à toutes les pages, forêts sombres, troubles du cœur, serments, sanglots, larmes et baisers, nacelles au clair de lune, rossignols dans les bosquets, messieurs braves comme des lions, doux comme des agneaux, vertueux comme on ne l'est pas, toujours bien mis, et qui pleurent comme des urnes ${ }^{38}$.

Comme on peut le voir, ce sont des romans romantiques à quatre sous qui ont inspiré à Emma ses aspirations à un amour idéal. On pourrait cependant penser que le caractère stéréotypé est ici dû au fait qu'Emma ne lit que des œuvres de mauvaise qualité. Mais Flaubert rajoute plus tard un passage sur la lecture de la "grande" littérature:

Emma fut intérieurement satisfaite de se sentir arrivée du premier coup à ce rare idéal des existences pâles, où ne parviennent jamais les cœurs médiocres. Elle se laissa donc glisser dans les méandres lamartiniens, écouta les harpes sur les lacs, tous les chants de cygnes mourants, toutes les chutes de feuilles, les vierges pures qui montent au ciel, et la voix de l'Éternel discourant dans les vallons ${ }^{39}$.

Le passage cité fait bien sûr allusion aux grands auteurs romantiques, une littérature "légitime" donc, qui est néanmoins, elle aussi, ramenée aux mêmes lieux communs et clichés que ceux déjà présents dans les lectures à quatre sous de l'enfance d'Emma.

On peut donc constater que chez Flaubert rien n'est plus à l'abri d'un soupçon généralisé d'inauthenticité, pas même la littérature légitime. Et un deuxième constat s'impose : Flaubert ne considère pas le mensonge et l'inauthenticité comme un simple phénomène limité au domaine de l'expression, mais pour lui, ceux-ci se manifestent également dans la conscience même. Dans l'acide chlorhydrique de cette analyse, la conscience d'Emma se décompose en une masse indistincte d'idées convenues. La conscience individuelle, chez saint Augustin encore zone de l'authenticité par excellence, est montrée comme envahie par des idées reçues, des stéréotypes et des

\footnotetext{
38 Flaubert, Madame Bovary, p. 48.

39 Ibid., p. 98 sq.
} 
lieux communs, de sorte que la frontière entre authenticité et mensonge se situe maintenant à l'intérieur de la conscience individuelle. De ce fait, Flaubert subvertit la base même ou bien la condition de possibilité de la véracité. Bien sûr il peut y avoir encore une expression authentique des opinions ou sentiments individuels, mais ce qui est inauthentique et mensonger, c'est cette conscience même, avant même qu'elle puisse s'articuler sur le mode du mensonge. Il s'ensuit que nous avons ici affaire à un type de mensonge qui ne relève plus de la volonté ou de l'incapacité d'expression d'un sujet, mais qu'il est devenu structurel ou bien « objectif » au sens de W. Benjamin.

Nathalie Sarraute, quant à elle, va plus loin encore dans la subversion entamée par Flaubert, car chez elle l'instance qui jusqu'alors avait servi de référence à toute théorie du mensonge, à savoir l'individu, se trouve être décomposée. Dans Le Planétarium, elle scrute ses personnages à travers le microscope d'une focalisation interne et du discours indirect libre. En agrandissant la conscience individuelle de cette manière, elle révèle que l'individu, qui de l'extérieur paraît comme un bloc homogène, est un être composé de plusieurs personnages. «Je » n'est pas seulement « un » Autre, mais « des » Autres, véritable caisse de résonance qui fait écho à différentes voix, à des lieux communs et des stéréotypes. Ainsi le «monologue» intérieur d'Alain, un des principaux personnages du roman, qui se sent tiraillé entre le milieu littéraire de l'écrivain Germaine Lemaire d'une part et sa famille bourgeoise d'autre part, est montré comme un véritable «polylogue», au cours duquel interviennent les voix de sa femme, de l'écrivaine célèbre et les lieux communs de tout le monde. Dans une conversation avec son idole, Alain construit un terrain d'entente entre eux en se servant de quelques clichés de la vie littéraire:

Rimbaud, Baudelaire et sa mère, le général Aupick... Paresseux, infantiles, gaspillant leur temps, perdant leur vie [...] Ce sont les modèles dont il veut qu'elle s'inspire. Elle lui obéit. Il regarde, ravi, en elle l'image à leur ressemblance $[\ldots]^{40}$.

Mais ce terrain d'entente sera miné ensuite, car leur conversation est interrompue par un appel téléphonique, après lequel Germaine change d'attitude: « Elle revient vers lui. Mais il sait que le spectacle est fini. [...] Il est rejeté, dégradé... Ah, c'est magnifique, cette supériorité... [... ${ }^{41} \gg$. Ce rejet le fait finalement revenir vers sa femme:

Gisèle... mon amour, ma femme... Ce nom exorcise. Le répéter sans fin... Gisèle... c'est l'apaisement, c'est la sécurité. C'est cela qui est vrai, qui est sain. Ils ont tout de même raison avec leurs vieilles conventions; c'est le reste qui est tout faux, de la camelote. Et lui, l'imbécile, l'infantile, jouant au révolté, faisant l'énervé ${ }^{42} \ldots$

Pour éviter des malentendus, il convient de préciser ici qu'il ne s'agit chez N. Sarraute ni de vilipender la bourgeoisie ni de critiquer le milieu littéraire, et surtout pas d'attaquer la soi-disant «mauvaise foi » de ses personnages ou bien leur mensonge à soi. Son approche est plus radicale, car elle montre que nous

\footnotetext{
${ }^{40}$ Nathalie Sarraute, Le Planétarium, Paris, Gallimard, 1972, p. 83.

${ }^{41}$ Ibid., p. 88.

${ }^{42}$ Ibid., p. 91.
} 
portons en nous une multiplicité de voix différentes et que ce que nous appelons notre « conviction » n'est souvent que la dominante parmi toute une chorale de voix dissonantes. Compte tenu de cette diversité, il faut se demander où se trouve par exemple la "véritable" personnalité d'Alain, et quand il est vraiment susceptible de "mentir": quand il est avec Germaine Lemaire pour dénigrer sa famille bourgeoise ou bien quand il se trouve en présence de sa femme pour critiquer les milieux littéraires? Ainsi, N. Sarraute, dont le premier roman a été salué par la préface d'un Jean-Paul Sartre enthousiaste, sape les fondements de l'authenticité même et montre que le règne du Man de Heidegger se prolonge jusque dans les régions les plus intimes de l'individu.

Cependant, le roman de Sarraute ne se limite pas à subvertir les fondements de la théorie classique du mensonge, il en modélise une autre conception où la forme joue un rôle considérable. Le Planétarium montre que l'idée selon laquelle nous avons d'abord une conviction qui est ancrée en nous et que nous essayons ensuite d'exprimer est erronée. Chez Sarraute, il n'y a pas de conviction qui précède toute expression, mais celle-ci se développe au cours de la communication ou bien dans le contact avec autrui. La conviction s'élabore donc grâce à l'expression et grâce à la conversation avec les autres. Comme Kleist l'a bien montré dans son essai intitulé Über die allmähliche Verfertigung der Gedanken beim Reden, nous développons nos convictions non pas indépendamment du discours, mais en parlant avec les autres ${ }^{43}$.

Il s'ensuit que la conviction ne peut plus servir comme point de départ intouchable de toute théorie du mensonge, il n'y a pas d'identité pure qui se construise dans un simple rapport à soi. Cela signifie-t-il que la notion même de mensonge serait devenue obsolète, car il n'y aurait plus d'aune à laquelle mesurer s'il y a oui ou non mensonge? Certainement pas, car il existe toujours une mesure du mensonge, mais celle-ci ne s'établit plus, comme dans la théorie classique, avant l'avènement de l'expression et en dehors de tout contact avec les autres, mais plutôt grâce à un acte performatif par lequel nous signifions aux autres que telle est notre opinion ou bien notre conviction. Notre conviction sera donc celle que nous aurons choisie après ce processus et que nous aurons signalée comme telle aux autres. Grâce à cet acte, nous nous attribuons nous-même une conviction d'après laquelle on peut bien sûr juger si nous exprimons ce que nous avons choisi et désigné comme notre conviction ou si nous mentons. Ainsi la théorie esthétique du mensonge ne met pas en question la notion même du mensonge mais en transforme les coordonnées.

${ }^{43}$ Heinrich von KLEIST, « Über die allmähliche Verfertigung der Gedanken beim Reden », in Heinrich von Kleist, Werke und Briefe in vier Bänden, vol. III, éd. par Siegfried Streller, commenté par Peter GoldAmmer, Frankfurt a. M., Insel, 1986, p. 722-723. 
